\title{
A drug-drug interaction study to assess the potential effect of acid-reducing agent, lansoprazole, on quizartinib pharmacokinetics
}

\author{
Jianke $\mathrm{Li}^{1}$ • Denise Trone ${ }^{1} \cdot$ Jeanne Mendell ${ }^{2}$ Patrick O'Donnell ${ }^{1} \cdot$ Natalie Cook ${ }^{1}$
}

Received: 10 May 2019 / Accepted: 26 July 2019 / Published online: 5 August 2019

(c) The Author(s) 2019

\begin{abstract}
Purpose Quizartinib, a potent, selective FMS-like tyrosine kinase 3 (FLT3) inhibitor, is currently in phase 3 development for patients with FLT3-internal tandem duplication-mutated acute myeloid leukemia (AML). Acid-reducing agents (ARAs; e.g., proton pump inhibitors) are frequently used during AML treatment. Since quizartinib demonstrates pH-dependent solubility, the effect of lansoprazole coadministration on pharmacokinetics (PK) of quizartinib tablet formulation was assessed. Methods An open-label, parallel-group study randomized 64 healthy adults to single-dose quizartinib $30 \mathrm{mg}$ alone (reference) or lansoprazole (60 mg once daily, days 1-5) + single-dose quizartinib $30 \mathrm{mg}$ (day 5) (test). Plasma concentrations of quizartinib and its active metabolite, AC886, were measured to $504 \mathrm{~h}$ postdose; the effect of lansoprazole on quizartinib PK was assessed by analysis of variance.

Results Quizartinib geometric mean ratios (test/reference) and 90\% confidence intervals for maximum observed plasma concentration $\left(C_{\max }\right)$, area under the concentration-time curve to last measurable drug concentration $\left(\mathrm{AUC}_{\text {last }}\right)$, and AUC to infinity were $86.11 \%(78.4 \%, 94.6 \%), 93.96 \%$ (79.6\%, 110.9\%), and 95.30\% (80.2\%, 113.3\%), respectively. Comparisons showed a modest decrease in quizartinib absorption when co-administered with lansoprazole, with lower limits for $C_{\max }$ and $\mathrm{AUC}_{\text {last }}$ just below $80-125 \%$ limits. Treatment-emergent adverse events were mild or moderate; the most frequent in either treatment group were headache [quizartinib alone: $(n=3) 10 \%$ ], upper respiratory tract infection [quizartinib alone: $(n=2)$ 6.7\%; lansoprazole + quizartinib: $(n=3) 9.1 \%$ ], and muscle tightness [quizartinib alone: $(n=2) 6.7 \%$ ].

Conclusions Concomitant lansoprazole had minimal effect on quizartinib PK as a formulated tablet, indicating that quizartinib can be administered with ARAs.
\end{abstract}

Keywords Quizartinib · Proton pump inhibitor · Gastric acid modifier · Drug interaction · AML

\section{Introduction}

The majority of patients with acute myeloid leukemia (AML) have poor prognosis despite recent advances in therapy because of disease heterogeneity driven by clonal

Jianke Li and Denise Trone were employees of Daiichi Sankyo, Inc., and Ambit Biosciences at the time this study was conducted.

Electronic supplementary material The online version of this article (https://doi.org/10.1007/s00280-019-03915-1) contains supplementary material, which is available to authorized users.

Jianke Li

jiankelius@yahoo.com

1 Daiichi Sankyo, Inc., 10201 Wateridge Circle, Suite 240, San Diego, CA 92121, USA

2 Daiichi Sankyo, Inc., Basking Ridge, NJ, USA evolution, which contributes to treatment resistance [1-3]. Patients with mutations in the internal tandem duplication (ITD) region of the FMS-like tyrosine kinase 3 (FLT3) gene, one of the most common driver mutations in AML, have particularly poor prognosis following conventional chemotherapy [1, 2, 4]. Quizartinib is an orally administered, highly potent and selective next-generation FLT3 inhibitor currently in phase 3 development in patients with FLT3-ITD-mutated AML (QuANTUM-R: NCT02039726; QuANTUM-First: NCT02668653). Quizartinib treatment in previous phase 2 trials demonstrated composite complete remission (CRc) rates of $44-47 \%$ in patients with relapsed or refractory FLT3-ITD-mutated AML, and $34-42 \%$ of patients were bridged to potentially curative hematopoietic stem cell transplant [5-7]. Quizartinib was generally well tolerated; the main adverse events (AEs) were manageable myelosuppression and QT prolongation that was 
mitigated with dose reduction $[5,7,8]$. Results from the completed QuANTUM-R phase 3 study in patients with relapsed/refractory FLT3-ITD-mutated AML, quizartinib (30-mg starting dose with escalation to $60 \mathrm{mg}$ once daily; $n=245$ ) showed prolonged overall survival versus standard chemotherapy ( $n=122$; median 6.2 months vs. 4.7 months, respectively; hazard ratio: $0.76 ; P=0.02$ ), with a greater proportion of patients undergoing hematopoietic stem cell transplantation (32\% vs. $11 \%$, respectively) [9].

Patients with AML often experience gastrointestinal AEs (i.e., diarrhea, nausea, and vomiting) with antileukemia treatment $[10,11]$, including quizartinib $[5,7,12]$. Acidreducing agents (ARAs), such as proton pump inhibitors (PPIs), may be administered as part of supportive care to patients with AML [13, 14]. In one assessment, approximately $20-33 \%$ of cancer patients received ARAs, of which PPIs were the most commonly prescribed [15]. PPIs increase intragastric $\mathrm{pH}$ and may subsequently reduce the solubility and bioavailability of oral tyrosine kinase inhibitors (TKIs) [16].

In vitro data indicate that quizartinib dihydrochloride has $\mathrm{pH}$-dependent solubility. It is important to understand factors influencing pharmacokinetics (PK) to ensure appropriate exposure to oral TKIs such as quizartinib. Previous PK studies in healthy subjects and patients with AML [12, 17] indicated that quizartinib reaches maximum concentrations at $4 \mathrm{~h}$ (range 2-8 h) and demonstrates dose-proportional PK at doses ranging from 20 to $90 \mathrm{mg}$, with an accumulation ratio of approximately 4 (data on file, Daiichi Sankyo Inc., Basking Ridge, NJ, USA). Quizartinib is metabolized to its pharmacologically active primary metabolite AC886, which has similar potency and selectivity as the parent [dissociation constant $(\mathrm{Kd})$ values for FLT3 of $1.3 \mathrm{nM}$ for quizartinib and $0.54 \mathrm{nM}$ for AC886], with a metabolite-parent ratio of $60 \%$ following repeated daily dosing in patients with AML (data on file, Daiichi Sankyo Inc., Basking Ridge, NJ, USA). Quizartinib and AC886 half-lives were estimated as $73 \mathrm{~h}$ and $119 \mathrm{~h}$, respectively, in AML patients (data on file, Daiichi Sankyo Inc., Basking Ridge, NJ, USA).

We report results from a phase 1 study that examined the effects of lansoprazole, a PPI, on the PK of quizartinib (in tablet formulation) and its active metabolite AC866.

\section{Methods}

\section{Study design}

This phase 1, open-label, randomized, parallel-group study was conducted at a single center in the USA from January to May 2015 to determine the effect of gastric $\mathrm{pH}$ modification by lansoprazole on the PK of quizartinib and its active metabolite, AC886, in healthy subjects. The tolerability and safety of quizartinib administered alone and in combination with lansoprazole were also assessed. The study was conducted in compliance with the Declaration of Helsinki and the International Conference on Harmonisation (ICH)/ Good Clinical Practice as well as all applicable state, local, and federal regulations. The study protocol, amendments, and informed consent forms were reviewed and approved by the institutional review board. All subjects provided written informed consent before any study-related procedure took place.

\section{Eligibility}

Healthy males and nonpregnant females, $18-55$ years of age, were eligible. Key inclusion criteria were body mass index of $18-32 \mathrm{~kg} / \mathrm{m}^{2}$; serum potassium, magnesium, and calcium within normal limits; adequate hepatic and coagulation parameters; and adequate renal function, as defined by serum creatinine $\leq 1.5 \times$ upper limit of normal and estimated creatinine clearance at screening $\geq 80 \mathrm{~mL} / \mathrm{min}$ according to the Cockcroft-Gault equation. Main exclusion criteria were history of clinically significant disease, abnormality, or drug allergy; treatment with any investigational product in a clinical study within 30 days (or five drug-half-lives, whichever was longer); and use or anticipated use of prescription medications including hormonal contraceptives, over-the-counter medications, herbal products, or dietary supplements. Subjects who received drugs with a risk of QT prolongation or torsade de pointes within 14 days of study initiation were excluded.

\section{Randomization and treatments}

Subjects were randomized into two groups: lansoprazole and quizartinib (lansoprazole + quizartinib; test group) and quizartinib alone (reference group). On days 1-5, subjects in the test group were administered 60-mg lansoprazole ( $2 \times 30 \mathrm{mg}$ oral delayed-released capsule) once daily in the morning before food. On day 5 , subjects in both groups received quizartinib as a single dose in tablet form containing 30-mg quizartinib dihydrochloride (equivalent to 26.5mg quizartinib free-base) after a 10-h fast and continued fasting for at least $4 \mathrm{~h}$ after dosing. All study drugs were taken with approximately $240 \mathrm{~mL}$ of water. Test group subjects received the 60-mg lansoprazole dose concomitantly with quizartinib on day 5 .

The study drug doses were selected because they were considered appropriate to assess a maximum potential drug-drug interaction between quizartinib and lansoprazole based on the starting dose for quizartinib in the pivotal phase 3 study (QuANTUM-R, NCT02039726), and the highest daily lansoprazole dose of $60 \mathrm{mg}$ received by AML patients in a quizartinib phase 2 study (NCT00989261). For this 
study, $30 \mathrm{mg}$ was selected due to safety considerations in healthy volunteers, and $30 \mathrm{mg}$ was the highest dose strength for the tablet formulation.

\section{Sample collection and pharmacokinetic analyses}

Blood samples for measurement of plasma quizartinib and AC886 concentrations were collected from all subjects before quizartinib dosing on day 5 and at $0.25,0.5,1,2,3,4$, $5,6,8,12,24,36,48,72,96,120,144,168,192,216,288$, 360,432 , and $504 \mathrm{~h}$ after quizartinib administration. Plasma concentrations were determined by BASi (West Lafayette, IN, USA) using a validated analytical method.

The PK population consisted of all subjects who received the quizartinib dose and had evaluable maximum observed plasma concentration $\left(C_{\max }\right)$ or area under the plasma concentration-time curve from time 0 to the last quantifiable plasma concentration $\left(\mathrm{AUC}_{\text {last }}\right)$ or from time 0 extrapolated to infinity $\left(\mathrm{AUC}_{\mathrm{inf}}\right)$ for quizartinib, $\mathrm{AC} 886$, or quizartinib + AC886, and did not have any significant protocol deviations. PK parameters in plasma for quizartinib, AC886, and quizartinib + $\mathrm{AC} 886$, including $C_{\max }, \mathrm{AUC}_{\text {last }}, \mathrm{AUC}_{\text {inf }}$, terminal elimination half-life $\left(T_{1 / 2}\right)$, time to $C_{\max }\left(T_{\max }\right)$, apparent systemic clearance (CL/F), and apparent volume of distribution $\left(V_{\mathrm{z}} / F\right)$, were calculated using WinNonlin ${ }^{\circledR}$ version 6.4 (Certara USA, Inc., Princeton, NJ, USA). In PK analyses of quizartinib and $\mathrm{AC} 886$, concentration values that were below the limit of quantification (BLQ) were set to zero unless flanked by measurable concentrations, in which case they were set to missing. In PK analyses of quizartinib + AC886, concentration values that were BLQ for both quizartinib and $\mathrm{AC} 886$ were set to zero. If $\mathrm{AUC}_{\mathrm{inf}}$ could not be determined for quizartinib and $\mathrm{AC} 886$, then $\mathrm{AUC}_{\text {inf }}$ was not reported for quizartinib + AC886.

\section{Bioanalytic methods}

Quizartinib and AC886 plasma concentrations were measured by BASi (Bioanalytical Systems Inc., West Lafayette, IN, USA) using a validated liquid chromatography (LC)-tandem mass spectrometry (MS) method. Quizartinib and AC886 were extracted from $\mathrm{K}_{2}$ EDTA human plasma by solid-phase-supported liquid extraction with methyl tert-butyl ether; $\mathrm{d}_{4}$-quizartinib and $\mathrm{d}_{4}$-AC886 were added as internal standards prior to extraction. Samples were injected into an LC-MS/MS system (LC: Nexera System, Shimadzu Scientific Instruments, Columbia, MD, USA; MS: API 5500 ${ }^{\mathrm{TM}}$, SCIEX, Framingham, MA, USA) using a PFP column (Phenomenex/Kinetex, Torrance, CA, USA) with a gradient ammonium formate/formic acid/acetonitrile/water mobile phase. The monitored mass-to-charge $(\mathrm{m} / \mathrm{z})$ values for the precursor ions were 561.1, 577.1, 565.1, and 581.1 for quizartinib, AC886, $\mathrm{d}_{4}$-quizartinib, and $\mathrm{d}_{4}$-AC886, respectively. The $\mathrm{m} / \mathrm{z}$ values monitored for the product ions were 421.1 for quizartinib and AC886, and 425.1 for $\mathrm{d}_{4}$-quizartinib and $\mathrm{d}_{4}$-AC886. For both quizartinib and AC886, the analytical range validated was from 0.50 to $500 \mathrm{ng} / \mathrm{mL}$. For quizartinib at the lower limit of quantitation (LLOQ), precision was $5.2 \%$ coefficient of variation (CV) and accuracy was $1.0 \%$ bias across 16 runs; accuracy ranged from -10.2 to $7.8 \%$ bias within runs. For quizartinib at the upper limit of quantitation, precision was $3.5 \% \mathrm{CV}$ and accuracy was $-1.6 \%$ bias across 16 runs; accuracy ranged from -6.6 to $6.4 \%$ bias within runs. For AC886 at the LLOQ, precision was $6.0 \% \mathrm{CV}$ and accuracy was $0.2 \%$ bias across 14 runs; accuracy ranged from -7.9 to $10.6 \%$ bias within runs. For $\mathrm{AC} 886$ at the upper limit of quantitation, precision was $2.9 \% \mathrm{CV}$ and accuracy was $-1.4 \%$ bias across 14 runs; accuracy ranged from -6.4 to $4.6 \%$ bias within runs.

\section{Safety analysis}

The safety analysis population consisted of all randomized subjects who received quizartinib or at least one dose of lansoprazole. Safety was assessed with physical examinations, vital signs, AE evaluations, 12-lead electrocardiograms (ECGs), and clinical laboratory tests (hematology, chemistry, and urinalysis). AEs were evaluated during the study according to the Medical Dictionary for Regulatory Activities version 17.1 and assessed for severity and relation to study drugs. Laboratory results were summarized based on National Cancer Institute Common Terminology Criteria for Adverse Events version 4.03.

As quizartinib was associated with QTcF (QT interval corrected with Fridericia's formulation) prolongation in an earlier study [12], ECGs were performed on all subjects at screening and on days -1 and 5. On day 5, ECGs were obtained before quizartinib administration and at 2, 3, 4, and $8 \mathrm{~h}$ after quizartinib administration. Analyses of QTcF interval data were based on ICH E14 categories [18].

\section{Statistics}

A sample size of 25 subjects per group was determined to yield a $\leq 10 \%$ relative standard error of the mean, based on the observed intersubject coefficient of variation $(\mathrm{CV} \%)$ of approximately $60 \%$ for PK parameters in a previous drug-drug interaction study in healthy volunteers (data on file, Daiichi Sankyo, Inc., Basking Ridge, NJ, USA). Up to 32 subjects/group were planned to account for possible premature withdrawal. Descriptive statistics were used to summarize plasma concentrations and PK parameters of the PK analysis population. Safety parameters were summarized in the safety analysis population using descriptive statistics. All descriptive statistics were calculated using SAS ${ }^{\circledR}$ software version 9.3 (SAS Institute Inc., Cary, NC, USA). The effects 
of lansoprazole on PK of quizartinib, AC886, and quizartinib + $\mathrm{AC} 886$ were assessed by comparing $C_{\max }, \mathrm{AUC}_{\mathrm{inf}}$, and $\mathrm{AUC}_{\text {last }}$ of the two treatment arms using an analysis of variance (ANOVA) model with treatment as a fixed effect. PK parameters were natural logarithm (ln)-transformed prior to analyses. The ln-transformed PK results were backtransformed by exponentiation to obtain the geometric least squares mean (LSM) for each treatment and calculate geometric LSM ratios for pairwise comparisons. Absence of a drug-drug interaction was concluded if the $90 \%$ confidence intervals (CIs) of geometric LSM ratios (test group:reference group) for $C_{\max }$ and AUC were completely contained within the accepted $80-125 \%$ interval, in accordance with regulatory agency guidelines $[19,20]$.

\section{Results}

\section{Demographics and baseline characteristics}

A total of 64 subjects were enrolled, with 31 randomized to the quizartinib-alone group and 33 to the lansoprazole + quizartinib group (Online Resource 1). Overall, five subjects did not complete the study. One subject from the quizartinib-alone group was withdrawn from the study prior to dosing because of a low platelet count and was excluded from the safety population; four subjects from the lansoprazole + quizartinib group withdrew consent. Demographic and baseline characteristics of the safety population were generally similar between treatment groups (Table 1). The groups were balanced with respect to age, sex, weight, and body mass index. The racial composition differed somewhat between groups, with the test group having a lower percentage of white subjects (54.5\% vs. $66.7 \%$ ) and a higher percentage of black (30.3\% vs. $23.3 \%)$ and "other" $(15.2 \%$ vs. $6.7 \%)$ subjects.

\section{Pharmacokinetic results}

The mean plasma concentration-time profiles of quizartinib were similar between the two treatment groups (Fig. 1a, b). Plasma PK parameters for quizartinib are summarized in Table 2. Median $T_{\max }$ was $3.5 \mathrm{~h}$ with lansoprazole + quizartinib and $4.0 \mathrm{~h}$ with quizartinib alone. Geometric mean $C_{\max }$ values were slightly lower after coadministration of lansoprazole + quizartinib than after quizartinib alone (90.3 vs. $105 \mathrm{ng} / \mathrm{mL}$, respectively); $C_{\max }$ values demonstrated low variability for both treatments, with $\mathrm{CV}$ of $27.2 \%$ and $16.3 \%$, respectively. Similarly, geometric mean $\mathrm{AUC}_{\text {last }}$ and $\mathrm{AUC}_{\text {inf }}$ were also slightly lower for lansoprazole + quizartinib than for quizartinib alone ( $\mathrm{AUC}_{\text {last }}: 7830 \mathrm{vs} .8330 \mathrm{ng} \mathrm{h} /$ $\mathrm{mL}$; $\mathrm{AUC}_{\text {inf }}: 8260$ vs. $8660 \mathrm{ng} \mathrm{h} / \mathrm{mL}$, respectively); AUC values demonstrated medium variability in both groups (CV 45.0-46.4\% with lansoprazole + quizartinib; CV 35.2-37.4\% with quizartinib alone). Geometric mean $\mathrm{CL} / F$ values were comparable between treatment groups $(3.2 \mathrm{~L} / \mathrm{h}$ with lansoprazole + quizartinib and $3.1 \mathrm{~L} / \mathrm{h}$ with quizartinib alone); geometric mean $V_{z} / F$ values for quizartinib were slightly higher for lansoprazole + quizartinib compared with quizartinib alone (479 and $431 \mathrm{~L}$, respectively). Geometric mean $T_{1 / 2}$ was comparable between groups ( $107.4 \mathrm{~h}$ for lansoprazole + quizartinib and $102.2 \mathrm{~h}$ for quizartinib alone).

\section{Effect of lansoprazole on quizartinib PK}

Quizartinib exposure decreased slightly after repeated lansoprazole administration. Quizartinib $C_{\max }, \mathrm{AUC}_{\text {last }}$, and $\mathrm{AUC}_{\text {inf }}$ were $13.9 \%, 6.0 \%$, and $4.7 \%$ lower, respectively,
Table 1 Demographics and baseline characteristics of subjects in the study

\begin{tabular}{lclc}
\hline & Quizartinib, $n=30^{\mathrm{a}}$ & $\begin{array}{l}\text { Lansoprazole + quizarti- } \\
\text { nib, } n=33\end{array}$ & Overall, $n=63$ \\
\hline $\begin{array}{l}\text { Age, years, mean (SD) } \\
\text { Sex, } n(\%)\end{array}$ & $36.4(9.1)$ & $32.9(9.0)$ & $34.6(9.1)$ \\
$\begin{array}{l}\text { Female } \\
\text { Male }\end{array}$ & $13(43.3)$ & $14(42.4)$ & $27(42.9)$ \\
Race, $n(\%)$ & $17(56.7)$ & $19(57.6)$ & $36(57.1)$ \\
White & $20(66.7)$ & $18(54.5)$ & $38(60.3)$ \\
Black & $7(23.3)$ & $10(30.3)$ & $17(27.0)$ \\
Asian & $1(3.3)$ & 0 & $1(1.6)$ \\
Other & $2(6.7)$ & $5(15.2)$ & $7(11.1)$ \\
Weight, kg, mean (SD) & $74.7(10.0)$ & $75.7(11.4)$ & $75.2(10.7)$ \\
Body mass index, $\mathrm{kg} / \mathrm{m}^{2}$, mean \\
(SD) & $25.9(3.2)$ & $25.8(2.8)$ & $25.8(3.0)$ \\
\hline
\end{tabular}

$S D$ standard deviation

${ }^{a}$ One subject in the quizartinib-alone group was withdrawn due to low platelet count on day 4 before receiving quizartinib and was not included 
Fig. 1 Mean ( \pm standard deviation) concentration-time profiles of quizartinib in plasma after administration of a single 30-mg dose of quizartinib alone or with $2 \times 30 \mathrm{mg}$ lansoprazole (linear scale) from time zero to $504 \mathrm{~h} \mathrm{(a)} \mathrm{and} \mathrm{from} \mathrm{time} \mathrm{zero} \mathrm{to}$ $12 \mathrm{~h}$ (b)
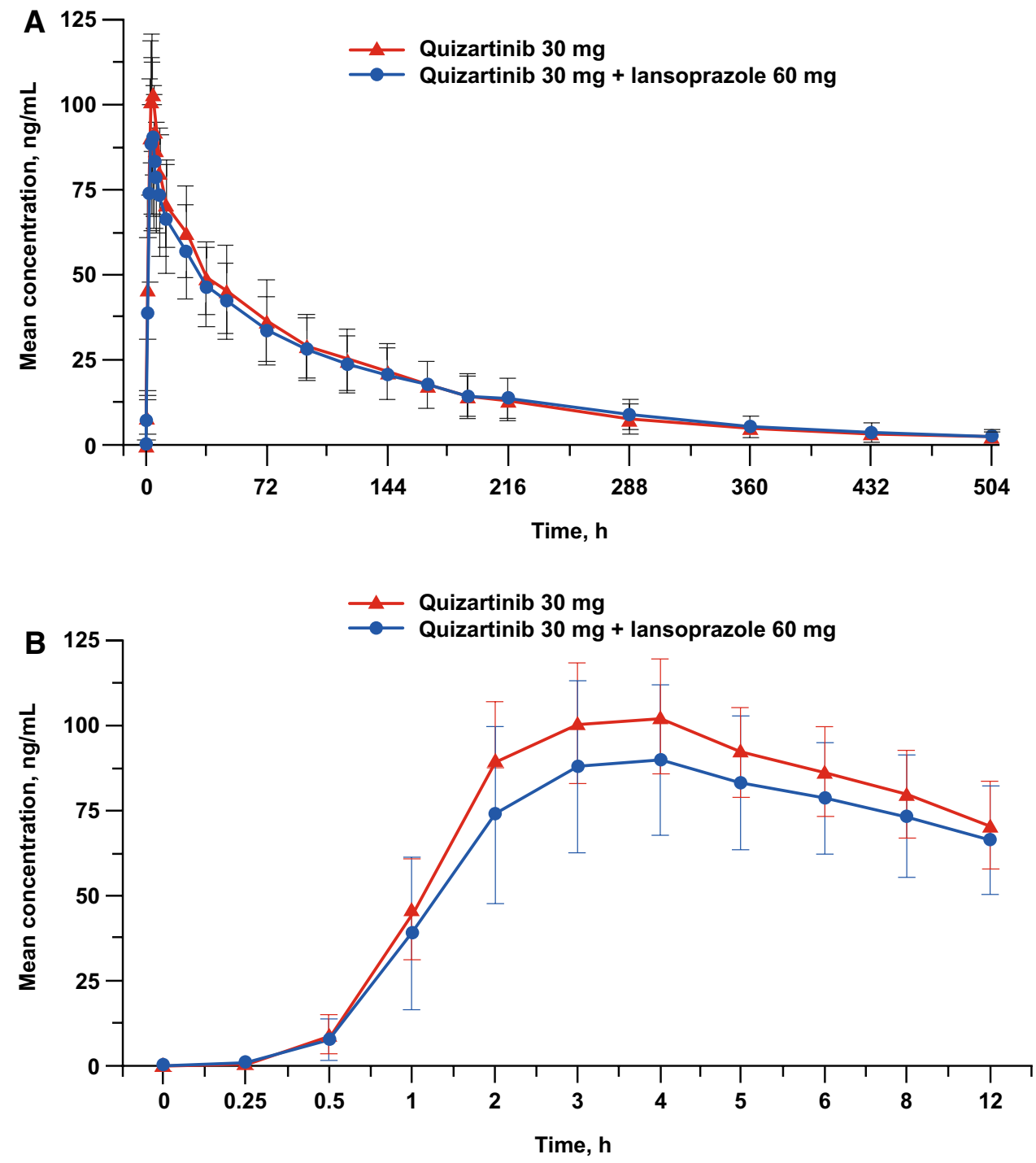

for lansoprazole + quizartinib than for quizartinib alone (Table 3). The bounds of the $90 \% \mathrm{CI}$ for $C_{\max }(78.4 \%$, $94.6 \%)$ and $\mathrm{AUC}_{\text {last }}(79.6 \%, 110.9 \%)$ were slightly outside of the accepted 80-125\% interval for establishing an absence of a significant drug-drug interaction, while the $90 \%$ CI for $\mathrm{AUC}_{\text {inf }}(80.2 \%, 113.3 \%)$ fell within the accepted range.

\section{Effect of lansoprazole on AC886 and quizartinib + AC886 PK}

Geometric LSMs for AC886 $C_{\text {max }}, \mathrm{AUC}_{\text {last }}$, and $\mathrm{AUC}_{\mathrm{inf}}$ decreased after lansoprazole exposure, by approximately $23 \%, 25 \%$, and $18 \%$, respectively (Table 4 ). The PK profiles of AC886 in the two treatment groups were similar, with comparable $T_{\max }(6.0 \mathrm{~h}$ with lansoprazole + quizartinib and $5.0 \mathrm{~h}$ with quizartinib) and $T_{1 / 2}$ (101.0 $\mathrm{h}$ with lansoprazole + quizartinib and $98.3 \mathrm{~h}$ with quizartinib). The quizartinib + AC886 geometric LSMs for $C_{\text {max }}, \mathrm{AUC}_{\text {last }}$, and AUC inf were also lowered by approximately $15 \%, 8 \%$, and $7 \%$, respectively, after lansoprazole treatment (Table 5). Median $T_{\max }$ was $4.0 \mathrm{~h}$ in both treatment groups.

\section{Safety}

Quizartinib administered with or without lansoprazole was generally safe and well tolerated by healthy subjects. Twenty subjects $[31.7 \% ; 9(30.0 \%)$ in the quizartinib-alone group and $11(33.3 \%)$ in the lansoprazole + quizartinib group] experienced at least one treatment-emergent AE (TEAE) following quizartinib treatment on day 5. The most common TEAEs occurring in either treatment group were headache (3 subjects in the quizartinib-alone group, 10\%), upper respiratory tract infection [2 (6.7\%) and $3(9.1 \%)$ subjects in the quizartinib-alone and lansoprazole + quizartinib groups, respectively], and muscle tightness ( 2 subjects in the quizartinib-alone group, 6.7\%) (Online Resource 2). Of 
Table 2 Plasma PK parameters of quizartinib administered with and without lansoprazole

\begin{tabular}{lcc}
\hline PK parameter & Quizartinib, $n=30$ & $\begin{array}{l}\text { Lansopra- } \\
\text { zole + quizartinib, } \\
n=32\end{array}$ \\
\hline Median $T_{\max }(\min , \max ), \mathrm{h}$ & $4.0(2.0,8.0)$ & $3.5(2.0,8.0)$ \\
$C_{\max }(\mathrm{CV} \%), \mathrm{ng} / \mathrm{mL}^{\mathrm{a}}$ & $105(16.3)$ & $90.3(27.2)$ \\
$\mathrm{AUC}_{\text {last }}(\mathrm{CV} \%), \mathrm{ng} \mathrm{h} / \mathrm{mL}^{\mathrm{a}}$ & $8330(35.2)$ & $7830(45.0)$ \\
$\mathrm{AUC}_{\text {inf }}(\mathrm{CV} \%), \mathrm{ng} \mathrm{h} / \mathrm{mL}^{\mathrm{a}}$ & $8660(37.4)$ & $8260(46.4)^{\mathrm{b}}$ \\
$\mathrm{Mean}_{1 / 2}(\mathrm{SD}), \mathrm{h}^{\mathrm{c}}$ & $102.2(29.0)$ & $107.4(28.0)^{\mathrm{b}}$ \\
$\mathrm{CL} / F(\mathrm{CV} \%), \mathrm{L} / \mathrm{h}^{\mathrm{a}}$ & $3.1(37.4)$ & $3.2(46.4)^{\mathrm{b}}$ \\
$V_{\mathrm{z}} / F(\mathrm{CV} \%), \mathrm{L}^{\mathrm{a}}$ & $431(28.4)$ & $479(39.0)^{\mathrm{b}}$ \\
\hline
\end{tabular}

$A U C_{i n f}$ area under the concentration-time curve from time 0 to infinity, $A U C_{\text {last }}$ area under the concentration-time curve from time 0 to the time of the last quantifiable concentration, $C L / F$ apparent clearance, $C_{\max }$ maximum observed concentration, $C V$ coefficient of variation, $P K$ pharmacokinetic, $S D$ standard deviation, $T_{1 / 2}$ apparent terminal phase elimination half-life, $T_{\max }$ actual sampling time to reach maximum observed concentration, $V / F$ apparent volume of distribution in the terminal phase

${ }^{\mathrm{a}}$ Geometric mean

${ }^{\mathrm{b}} n=31$

${ }^{\mathrm{c}}$ Arithmetic mean (SD) the 20 subjects who experienced a TEAE, the majority (19 subjects; $95 \%$ ) reported TEAEs of mild severity. One subject in the quizartinib-alone group experienced headache of moderate severity that was considered possibly related to quizartinib. Three subjects experienced at least one quizartinib-related TEAE, including nausea, diarrhea, dizziness, headache, and fatigue. None of these quizartinib-related TEAEs occurred in more than one subject in either treatment group. There were no severe or serious TEAEs in this study.

No significant abnormalities in vital signs, hematology, or clinical chemistry were observed during the study. Most subjects in the quizartinib-alone and lansoprazole + quizartinib groups had a maximum $\mathrm{QTcF}$ value of $\leq 450 \mathrm{~ms}$ and a maximum change from baseline of $\leq 30 \mathrm{~ms}$. Two $(6.7 \%)$ and zero subjects in the quizartinib-alone and lansoprazole + quizartinib groups, respectively, had maximum QTcF $>450 \mathrm{~ms}$ but $\leq 480 \mathrm{~ms}$. None of the subjects had a maximum QTcF value $>480 \mathrm{~ms}$.
Table 3 Statistical comparisons (ANOVA) of quizartinib PK parameters after a single $30-\mathrm{mg}$ dose of quizartinib alone or with lansoprazole

\begin{tabular}{lccll}
\hline PK parameter & $\begin{array}{l}\text { Quizartinib } \\
\text { Geometric LS } \\
\text { mean, } n=30\end{array}$ & $\begin{array}{l}\text { Lansoprazole + quizartinib } \\
\text { Geometric LS mean, } n=32\end{array}$ & $\begin{array}{l}\text { Ratio of geomet- } \\
\text { ric LS mean, \% }\end{array}$ & $\begin{array}{l}\text { 90\% CI for ratio of } \\
\text { geometric LS mean, } \\
\%\end{array}$ \\
\hline$C_{\text {max }}, \mathrm{ng} / \mathrm{mL}$ & 104.8 & 90.3 & 86.11 & $78.36,94.64$ \\
$\mathrm{AUC}_{\text {last }}, \mathrm{ng} \mathrm{h} / \mathrm{mL}$ & 8328.9 & 7825.6 & 93.96 & $79.63,110.86$ \\
$\mathrm{AUC}_{\text {inf }}, \mathrm{ng} \mathrm{h} / \mathrm{mL}$ & 8664.7 & $8257.4^{\mathrm{b}}$ & 95.30 & $80.16,113.30$ \\
\hline
\end{tabular}

$A N O V A$ analysis of variance, $A U C_{i n f}$ area under the concentration-time curve from time 0 to infinity, $A U C$ last area under the concentration-time curve from time 0 to the time of the last quantifiable concentration, $C I$ confidence interval, $C_{\max }$ maximum observed concentration, $L S$ least squares, $P K$ pharmacokinetic

${ }^{\mathrm{a}}$ (Lansoprazole + quizartinib)/(quizartinib alone)

${ }^{\mathrm{b}} n=31$

Table 4 Statistical comparisons (ANOVA) of AC886 PK parameters after a single 30-mg dose of quizartinib alone or with lansoprazole

\begin{tabular}{lllll}
\hline PK parameter & $\begin{array}{l}\text { Quizartinib } \\
\text { Geometric LS mean, } \\
n=30\end{array}$ & $\begin{array}{l}\text { Lansoprazole + quizartinib } \\
\text { Geometric LS mean, } n=32\end{array}$ & $\begin{array}{l}\text { Ratio of geometric LS } \\
\text { mean, } \%^{\text {a }}\end{array}$ & $\begin{array}{l}\text { 90\% CI for ratio of } \\
\text { geometric LS mean, } \\
\%\end{array}$ \\
\hline$C_{\text {max }}, \mathrm{ng} / \mathrm{mL}$ & 21.2 & 16.3 & 76.93 & $57.13,103.60$ \\
$\mathrm{AUC}_{\text {last }}, \mathrm{ng} \mathrm{h} / \mathrm{mL}$ & 2778.7 & 2088.3 & 75.15 & $61.09,92.45$ \\
$\mathrm{AUC}_{\mathrm{inf}}, \mathrm{ng} \mathrm{h} / \mathrm{mL}$ & $2847.8^{\mathrm{b}}$ & $2329.3^{\mathrm{b}}$ & 81.79 & $67.50,99.11$ \\
${\text { Mean } T_{1 / 2}(\mathrm{SD}), \mathrm{h}}_{\text {Median } T_{\max }(\min , \max ), \mathrm{h}}$ & $58.3(26.8)^{\mathrm{b}}$ & $101.0(30.6)^{\mathrm{b}}$ & & \\
\hline
\end{tabular}

$A N O V A$ analysis of variance, $A U C_{i n f}$ area under the concentration-time curve from time 0 to infinity, $A U C_{\text {last }}$ area under the concentration-time curve from time 0 to the time of the last quantifiable concentration, $C I$ confidence interval, $C_{\max }$ maximum observed concentration, $L S$ least squares, $P K$, pharmacokinetic, $S D$ standard deviation, $T_{1 / 2}$ apparent terminal phase elimination half-life, $T_{\max }$ time to reach maximum plasma concentration

a (Lansoprazole + quizartinib)/(quizartinib)

${ }^{\mathrm{b}} n=29$ 
Table 5 Statistical comparisons (ANOVA) of quizartinib + AC886 PK parameters after a single 30-mg dose of quizartinib alone or with lansoprazole

\begin{tabular}{lllll}
\hline PK parameter & $\begin{array}{l}\text { Quizartinib } \\
\text { Geometric LS mean, } \\
n=30\end{array}$ & $\begin{array}{l}\text { Lansoprazole + quizartinib } \\
\text { Geometric LS mean, } n=32\end{array}$ & $\begin{array}{l}\text { Ratio of geometric LS } \\
\text { mean, \% }\end{array}$ & $\begin{array}{l}\text { 90\% CI for ratio of } \\
\text { geometric LS mean, } \\
\%\end{array}$ \\
\hline$C_{\text {max }}, \mathrm{ng} / \mathrm{mL}$ & 127.3 & 108.2 & 84.99 & $77.42,93.30$ \\
$\mathrm{AUC}_{\text {last }}, \mathrm{ng} \mathrm{h} / \mathrm{mL}$ & $11,525.3$ & $10,561.8$ & 91.64 & $83.84,100.16$ \\
$\mathrm{AUC}_{\text {inf }}, \mathrm{ng} \mathrm{h} / \mathrm{mL}$ & $12,012.5^{\mathrm{b}}$ & $11,127.3^{\mathrm{b}}$ & 92.63 & $83.94,102.22$ \\
Median $T_{\max }(\min , \max ), \mathrm{h}$ & $4.0(2.0,8.0)$ & $4.0(3.0,8.0)$ & & \\
\hline
\end{tabular}

$A N O V A$ analysis of variance, $A U C_{\text {inf }}$, area under the concentration-time curve from time 0 to infinity, $A U C_{\text {last }}$ area under the concentration-time curve from time 0 to the time of the last quantifiable concentration, $C I$ confidence interval, $C_{\max }$ maximum observed concentration, $L S$ least squares, $P K$ pharmacokinetic, $T_{\max }$ time to reach maximum plasma concentration

a (Lansoprazole + quizartinib)/(quizartinib)

${ }^{\mathrm{b}} n=29$

\section{Discussion}

Patients with AML often experience gastrointestinal complications, which may result in concomitant use of ARAs (such as PPIs, antacids, and $\mathrm{H} 2$ blockers) as a part of disease management [13]. However, concomitant use of ARAs may compromise the therapeutic benefit of oral TKIs, potentially through altered solubility and bioavailability. Although many factors may influence TKI absorption, one major determinant is $\mathrm{pH}$-dependent solubility [16]. A chemical structure analysis of 39 oral targeted cancer therapies in clinical development identified at least 11 compounds (28\%) predicted to have $\mathrm{pH}$-dependent solubility [15]. Of recently approved orally administered cancer therapeutics, $>50 \%$ are characterized as having $\mathrm{pH}$-dependent solubility. Clinical experience also indicates drug-drug interaction between PPIs and some TKIs, such as nilotinib or dasatinib [21-23]. In vitro assessment of quizartinib dihydrochloride confirmed the compound's solubility was pH dependent, with low solubility $\left(<4.0 \times 10^{-4} \mathrm{mg} / \mathrm{mL}\right)$ at physiological $\mathrm{pH}$ in the intestine. Thus, it was important to examine potential effects of a PPI (the most potent and long-lasting ARA class) on the bioavailability of the tablet formulation of quizartinib to ensure consistent plasma exposure and clinical efficacy and to inform dosing guidance. The metabolite, AC886, is active with similar pharmacology to quizartinib; thus, the total of parent and metabolite represents the pharmacologically active exposure.

PPIs are considered the most effective gastric ARAs, inhibiting acid secretion by as much as $90 \%$ and increasing gastric $\mathrm{pH}$ to $>4$ [24]. Lansoprazole is believed to be transformed into two active species at the secretory surface of the gastric parietal cell that are not present in the systemic circulation [25]. Although the elimination half-life of lansoprazole is less than $2 \mathrm{~h}$, an inhibitory effect on gastric acid secretion lasts over $24 \mathrm{~h}$. Following administration of lansoprazole $30 \mathrm{mg}$ once daily for 5 days, mean 24 -h intragastric
$\mathrm{pH}$ increased to 4.9 from a baseline of 2.1 ; thus, lansoprazole $60 \mathrm{mg}$ once daily was chosen for this study. This clinical study of the quizartinib tablet showed that increased gastric $\mathrm{pH}$ with lansoprazole had only a modest effect on the rate and extent of quizartinib absorption, with only $~ 15 \%$ mean decrease in $C_{\max }$ of quizartinib + AC886 and similar median $T_{\max }$ with coadministration of the tablet formulation of quizartinib and lansoprazole in healthy subjects. Variability in $C_{\max }$ values was slightly higher when lansoprazole was coadministered. Plasma exposure of quizartinib and total exposure (quizartinib $+\mathrm{AC} 886$ ), as assessed by $C_{\max }$ and AUCs, decreased slightly after repeated lansoprazole administration. In vitro, quizartinib solubility decreases with increase in $\mathrm{pH}$; thus, it is likely that the quizartinib tablet solubility in the human gastrointestinal tract is reduced when lansoprazole is co-administered. In the tablet formulation, 2-hydroxypropy-beta-cyclodextrin was used as a solubilityenhancing agent and could have contributed to minimizing the effect of the acid-reducing agent. The reduced solubility may cause the increased variability in the absorption of quizartinib in the gastrointestinal tract, as reflected in the increased variability in $C_{\max }$. However, the small decrease in exposure observed was not expected to be clinically relevant.

Metabolite-parent ratios (AC886/quizartinib) for AUC last and $\mathrm{AUC}_{\text {inf }}$ were similar with and without lansoprazole, with geometric means ranging from 0.267 to 0.334 . $T_{1 / 2}$ for quizartinib and AC886 were similar in both treatment groups. Therefore, quizartinib can also be administered with other gastric ARAs such as antacids and $\mathrm{H} 2$ blockers without clinical consequence.

Quizartinib administered as a single dose alone or coadministered with lansoprazole was well tolerated in this study. The most common TEAEs that occurred with quizartinib treatment were headache, upper respiratory tract infection, and muscle tightness. Most of these events were mild in severity. Most subjects had maximum QTcF values of $\leq 450 \mathrm{~ms}$ and maximum change from baseline $\leq 30 \mathrm{~ms}$, 
with 2 subjects with maximum QTcF ranging from $>450$ to $\leq 480 \mathrm{~ms}$ (quizartinib-alone group). However, no subjects had a maximum $\mathrm{QTcF}$ value $>480 \mathrm{~ms}$.

In conclusion, although the quizartinib dihydrochloride drug substance has $\mathrm{pH}$-dependent solubility in vitro, the PPI lansoprazole had minimal effect on quizartinib PK. Therefore, quizartinib as a formulated tablet can be administered with or without ARAs such as PPIs, antacids, and $\mathrm{H} 2$ blockers.

Acknowledgements The authors wish to acknowledge the contributions of the Principal Investigator, Christine Hale, MD, formerly of Covance, for assistance in conducting this study; Orlando Bravo of BASi for analytical measurements; and Jeffrey Stark of Worldwide Clinical Trials for pharmacokinetic analysis. Medical editorial assistance was provided by Ada Ao-Baslock, PhD, Accuverus Inc., and Amy Zannikos, PharmD, Peloton Advantage, LLC, an OPEN Health company, and funded by Daiichi Sankyo.

\section{Compliance with ethical standards}

Conflict of interest Jianke $\mathrm{Li}$ and Denise Trone were employees of Daiichi Sankyo, Inc., and Ambit Biosciences at the time this study was conducted. Jeanne Mendell, Patrick O'Donnell, and Natalie Cook are employees of Daiichi Sankyo, Inc.

Open Access This article is distributed under the terms of the Creative Commons Attribution 4.0 International License (http://creativeco mmons.org/licenses/by/4.0/), which permits unrestricted use, distribution, and reproduction in any medium, provided you give appropriate credit to the original author(s) and the source, provide a link to the Creative Commons license, and indicate if changes were made.

\section{References}

1. Dohner H, Weisdorf DJ, Bloomfield CD (2015) Acute myeloid leukemia. N Engl J Med 373(12):1136-1152. https://doi. org/10.1056/NEJMra1406184

2. Dombret H, Gardin C (2016) An update of current treatments for adult acute myeloid leukemia. Blood 127(1):53-61. https://doi. org/10.1182/blood-2015-08-604520

3. Stein EM, Tallman MS (2016) Emerging therapeutic drugs for AML. Blood 127(1):71-78. https://doi.org/10.1182/blood-201507-604538

4. Kottaridis PD, Gale RE, Frew ME, Harrison G, Langabeer SE, Belton AA, Walker H, Wheatley K, Bowen DT, Burnett AK, Goldstone AH, Linch DC (2001) The presence of a FLT3 internal tandem duplication in patients with acute myeloid leukemia (AML) adds important prognostic information to cytogenetic risk group and response to the first cycle of chemotherapy: analysis of 854 patients from the United Kingdom Medical Research Council AML 10 and 12 trials. Blood 98(6):1752-1759

5. Levis MJ, Perl AE, Dombret H, Dohner H, Steffen B, Rousselot P, Martinelli G, Estey EH, Burnett AK, Gammon G, Trone D, Leo E, Cortes JE (2012) Final results of a phase 2 open-label, monotherapy efficacy and safety study of quizartinib (AC220) in patients with FLT3-ITD positive or negative relapsed/refractory acute myeloid leukemia after second-line chemotherapy or hematopoietic stem cell transplantation [abstract]. Blood 120:673
6. Russell N, Tallman MS, Goldberg S, Perl AE, Marie JP, Martinelli G, Larson RA, Schiller G, Trone D, Gammon G, Levis M, Cortes JE (2014) Quizartinib (AC220) in patients with FLT3-ITD(+) relapsed or refractory acute myeloid leukemia: final results of a randomized phase 2 study [poster]. In: Annual meeting of the European Hematology Association, Milan, Italy, June 12-15, 2014

7. Schiller GJ, Tallman MS, Goldberg SL, Perl AE, Marie JP, Martinelli G, Larson RA, Russell N, Trone D, Gammon G, Levis MJ, Cortes JE (2014) Final results of a rendomized phase 2 study showing the clinical benefit of quizartinib (AC220) in patients with FLT3-ITD positive relapsed or refractory acute myeloid leukemia [abstract]. J Clin Oncol 32(15(suppl)):7100

8. Levis MJ, Cortes JE, Gammon GM, Trone D, Kang D, Li J (2016) Laboratory and clinical investigations to identify the optimal dosing strategy for quizartinib (AC220) monotherapy in FLT3-Itdpositive $(+)$ relapsed/refractory $(\mathrm{R} / \mathrm{R})$ acute myeloid leukemia (AML) [abstract]. Blood 128:4042

9. Cortes J, Khaled S, Martinelli G, Perl AE, Ganguly S, Russell N, Kramer A, Dombret H, Hogge D, Jonas BA, Yu-Hung Leung A, Mehta P, Montesinos P, Radsak M, Sica S, Arunachalam M, Holmes M, Kobayashi K, Namuyinga R, Ge N, Yver A, Zhang Y, Levis MJ (2018) Quizartinib significantly prolongs overall survival in patients with FLT3-internal tandem duplication-mutated (MUT) relapsed/refractory AML in the phase 3, randomized, controlled Quantum-R trial [abstract LB2600]. In: Annual congress of the European Hematology Association, Stockholm Sweden, June 14-17, 2018

10. Konig H, Levis M (2014) Is targeted therapy feasible in acute myelogenous leukemia? Curr Hematol Malig Rep 9(2):118-127. https://doi.org/10.1007/s11899-014-0198-1

11. Lopez-Jimenez J, Martin-Ballesteros E, Sureda A, Uralburu C, Lorenzo I, del Campo R, Fernandez C, Calbacho M, GarciaBelmonte D, Fernandez G (2006) Chemotherapy-induced nausea and vomiting in acute leukemia and stem cell transplant patients: results of a multicenter, observational study. Haematologica 91(1):84-91

12. Cortes JE, Kantarjian H, Foran JM, Ghirdaladze D, Zodelava M, Borthakur G, Gammon G, Trone D, Armstrong RC, James J, Levis M (2013) Phase I study of quizartinib administered daily to patients with relapsed or refractory acute myeloid leukemia irrespective of FMS-like tyrosine kinase 3-internal tandem duplication status. J Clin Oncol 31(29):3681-3687. https://doi. org/10.1200/jco.2013.48.8783

13. National Comprehensive Cancer Network (2017) NCCN Clinical Practice Guidelines in Oncology: Palliative Care v1.2018. National Comprehensive Cancer Network. https://www.nccn.org/ professionals/physician_gls/pdf/palliative.pdf. Accessed 18 Sep 2018

14. Tosetti C, Nanni I (2017) Use of proton pump inhibitors in general practice. World J Gastrointest Pharmacol Ther 8(3):180-185. https ://doi.org/10.4292/wjgpt.v8.i3.180

15. Smelick GS, Heffron TP, Chu L, Dean B, West DA, Duvall SL, Lum BL, Budha N, Holden SN, Benet LZ, Frymoyer A, Dresser MJ, Ware JA (2013) Prevalence of acid-reducing agents (ARA) in cancer populations and ARA drug-drug interaction potential for molecular targeted agents in clinical development. Mol Pharm 10(11):4055-4062. https://doi.org/10.1021/mp400403s

16. van Leeuwen RWF, Jansman FGA, Hunfeld NG, Peric R, Reyners AKL, Imholz ALT, Brouwers J, Aerts JG, van Gelder T, Mathijssen RHJ (2017) Tyrosine kinase inhibitors and proton pump inhibitors: an evaluation of treatment options. Clin Pharmacokinet 56(7):683-688. https://doi.org/10.1007/s40262-016-0503-3

17. Sanga M, James J, Marini J, Gammon G, Hale C, Li J (2017) An open-label, single-dose, phase 1 study of the absorption, metabolism and excretion of quizartinib, a highly selective and potent 
FLT3 tyrosine kinase inhibitor, in healthy male subjects, for the treatment of acute myeloid leukemia. Xenobiotica 47(10):856869. https://doi.org/10.1080/00498254.2016.1217100

18. The clinical evaluation of QT/QTc interval prolongation and proarrhythmic potential for non-antiarrhythmic drugs E14 (2005) International conference on harmonisation of technical requirements for registration of pharmaceuticals for human use. https:// www.ich.org/fileadmin/Public_Web_Site/ICH_Products/Guide lines/Efficacy/E14/E14_Guideline.pdf. Accessed 6 Feb 2018

19. Guidance for industry. Drug interaction studies—study design, data analysis, implications for dosing, and labeling recommendations (2012) Food and Drug Administration web site. U.S. Department of Health and Human Services; Food and Drug Administration; Center for Drug Evaluation and Research, Rockville, MD

20. Guideline on the investigation of drug interactions (2012) European Medicines Agency. http://www.ema.europa.eu/docs/en_GB/ document_library/Scientific_guideline/2012/07/WC50012960 6.pdf. Accessed 18 Sep 2018

21. Pape E, Michel D, Scala-Bertola J, Schiestel T, Harle A, Bouchet S, Contet A, Pochon C, Gambier N (2016) Effect of esomeprazole on the oral absorption of dasatinib in a patient with Philadelphia-positive acute lymphoblastic leukemia. Br J Clin Pharmacol 81(6):1195-1196. https://doi.org/10.1111/bcp.12895
22. Yin OQ, Gallagher N, Fischer D, Demirhan E, Zhou W, Golor G, Schran H (2010) Effect of the proton pump inhibitor esomeprazole on the oral absorption and pharmacokinetics of nilotinib. J Clin Pharmacol 50(8):960-967. https://doi.org/10.1177/0091270009 346061

23. Kletzl H, Giraudon M, Ducray PS, Abt M, Hamilton M, Lum BL (2015) Effect of gastric $\mathrm{pH}$ on erlotinib pharmacokinetics in healthy individuals: omeprazole and ranitidine. Anticancer Drugs 26(5):565-572. https://doi.org/10.1097/cad.0000000000000212

24. Blum RA, Shi H, Karol MD, Greski-Rose PA, Hunt RH (1997) The comparative effects of lansoprazole, omeprazole, and ranitidine in suppressing gastric acid secretion. Clin Ther 19(5):1013-1023

25. Prevacid [package insert] (2012) Takeda Pharmaceuticals America, Inc., Deerfield

Publisher's Note Springer Nature remains neutral with regard to jurisdictional claims in published maps and institutional affiliations. 